Artigo

\title{
Black Carbon em Material Particulado nas Residências de Idosos na Região Metropolitana de São Paulo, Brasil
}

\author{
Bruna Segalin, Fabio L.T. Gonçalves, Adalgiza Fornaro \\ Departamento de Ciências Atmosféricas, Instituto de Astronomia, \\ Geofísica e Ciências Atmosféricas, Universidade de São Paulo, São Paulo, SP, Brasil
}

Recebido: 01/12/2015 - Aceito: 14/04/2016

\begin{abstract}
Resumo
O material particulado fino (MP) e o Black Carbon são dois dos piores poluentes atmosféricos, afetando a saúde humana. Apesar disso, não há dados na literatura sobre estes poluentes no interior de residências no Brasil. Nosso objetivo é analisar as partículas menores que $0,25 \mu \mathrm{m}\left(\mathrm{MP}_{0,25}\right)$ e o rBC no interior de residências de idosos na Região Metropolitana de São Paulo (RMSP), em que a poluição do ar tem sido um problema sério devido às emissões veiculares. Em 60 residências, em cada amostra de 24 h foram medidos a massa e o rBC por análise gravimétrica e refletância, respectivamente. As concentrações em massa do $\mathrm{MP}_{0,25}$ e do $\mathrm{rBC}$ foram avaliadas em função das condições meteorológicas e do tráfego. A massa média do $\mathrm{MP}_{0,25}$ e de $\mathrm{rBC}$ foram de 13,6 e 2,8 $\mu \mathrm{g} / \mathrm{m}^{3}$, respectivamente. $\mathrm{O} \mathrm{MP}_{0,25}$ ultrapassou a recomendação da OMS para $\mathrm{MP}_{2,5}$ em $11,7 \%$ das residências, sendo que $26,2 \%$ da massa do $\mathrm{MP}_{0,25}$ foi composta por rBC. Considerando que o rBC é um traçador das emissões veiculares e, é prejudicial para a saúde humana, é importante que este poluente seja considerado em termos de ações de políticas públicas de controle para a melhoria da qualidade do ar na RMSP.
\end{abstract}

Palavras-chave: poluição do ar, black carbon, material particulado fino, ambiente interno.

\section{Black Carbon in Particulate matter in Residences of Elderly People in the Metropolitan Area of São Paulo, Brazil}

\begin{abstract}
Fine particulate matter (PM) and Black Carbon are two of the worst air pollutants, affecting human health, mainly elderly. Despite this fact, there is no data in literature about these pollutants indoor dwellings in Brazil. This study aims to analyze PM smaller than $0.25 \mu \mathrm{m}\left(\mathrm{PM}_{0.25}\right)$ and the Black Carbon ( $\left.\mathrm{rBC}\right)$ indoor dwellings of elderly located in the Metropolitan Area of Sao Paulo (RMSP), in which air pollution has been a serious problem due to the vehicular emissions. In 60 indoor dwellings, each PM sample was obtained during 24 hours, being the mass and rBC measured by gravimetric analysis and reflectance, respectively. The mass of $\mathrm{PM}_{0,25}$ and $\mathrm{rBC}$ were evaluated in function of the traffic and meteorological conditions; being the average concentrations 13.7 and $2.8 \mu \mathrm{g} / \mathrm{m}^{3}$, respectively. The $\mathrm{PM}_{0.25}$ exceeded the guideline from the WHO threshold for $\mathrm{PM}_{2.5}$ in $11.7 \%$ of households. Around $26 \%$ of the $\mathrm{PM}_{2.5}$ consisted in $\mathrm{rBC}$, which was due to their proximity of the intense traffic routes. Considering that the $\mathrm{rBC}$ is a tracer of the vehicular emissions and it is harmful to the elderly health, it is important that this pollutant be considered in terms of public control actions for air quality improvement in RMSP.
\end{abstract}

Keywords: air pollution, black carbon, fine particulate matter, indoor.

\section{Introdução}

O material particulado (MP), partículas líquidas e sólidas suspensas no ar, é um dos componentes da poluição atmosférica que pode causar problemas de visibilidade, interferir na radiação solar que chega na superfície terres- tre, afetar as plantas e animais e, principalmente causar danos à saúde humana. O MP é classificado de acordo com seu diâmetro aerodinâmico e tem sido observada relação direta com a capacidade de penetração no trato respiratório. As partículas inaláveis, as quais também são material par-

Autor de correspondência: Bruna Segalin, segalin@model.iag.usp.br. 
ticulado, têm diâmetro menor que $10 \mu \mathrm{m}\left(\mathrm{MP}_{10}\right)$, sendo que o material particulado fino $\left(\mathrm{d}<2,5 \mu \mathrm{m}, \mathrm{MP}_{2,5}\right)$ pode atingir os alvéolos pulmonares (Arbex et al., 2012). As partículas ultrafinas $\left(\mathrm{d}<0,1 \mu \mathrm{m}-\mathrm{MP}_{0,1}\right)$ podem translocar do epitélio respiratório para a corrente sanguínea e a partir dela atingir qualquer órgão do corpo, tais como coração e cérebro, podendo causar efeitos adversos sobre a função cardíaca e a coagulação do sangue, assim como nas funções do sistema nervoso central (Kreyling et al., 2006).

O MP tem ampla composição química que varia tanto em espécies quanto em quantidade, conforme a localização das fontes que originam estas partículas. As regiões urbanizadas são as mais afetadas pela poluição do ar devido à variedade e grande número de fontes. Além disso, as condições meteorológicas podem favorecer ou não a sua formação, diluição, remoção e transporte. Na Região Metropolitana de São Paulo (RMSP) o inverno é seco, com ventos calmos, ocorrendo predominância de sistemas de alta pressão sobre o continente e inversões térmicas mais frequentes próximas à superfície o que favorece a maior concentração de poluentes na região. Durante o inverno, somado com menor quantidade de precipitação, as concentrações de partículas inaláveis podem alcançar valores mais altos que o padrão nacional de qualidade do $\operatorname{ar}\left(150 \mu \mathrm{g} / \mathrm{m}^{3}\right.$, média 24 h) e com maior frequência de ocorrências (Miranda e Andrade, 2005). Em conjunto com o aumento das concentrações de $\mathrm{MP}_{2,5}$ observa-se aumento do teor de Black Carbon (Miranda et al., 2012), sendo que determinadas condições atmosféricas podem favorecer a formação de novas partículas ultrafinas (Martins et al., 2010).

O Black Carbon (BC) é um dos principais componentes do $\mathrm{MP}_{2,5}$ e suas fontes predominantes são queima de combustíveis fósseis (principalmente diesel) para transporte, combustíveis sólidos para indústrias, queima residencial de madeira e carvão, queima a céu aberto de biomassa (agricultura, floresta e vegetação em geral) e centrais elétricas que utilizam óleo pesado ou carvão (Janssen et al., 2012; Bond et al., 2013). Consequentemente, o BC é um indicador de uma mistura de material em partículas geradas a partir de uma grande variedade de fontes de combustão e, quando presente na atmosfera pode influenciar as mudanças climáticas e causar efeitos danosos à saúde.

$\mathrm{O}$ BC também é considerando uma forçante climática, pois tem papel importante no clima da Terra, ele absorve a radiação solar aquecendo a atmosfera e dispersa a luz reduzindo a radiação solar que atinge a superfície e que é refletida de volta para o espaço (Bond et al., 2013). Além disso, o BC afeta os processos de nuvem alterando o número de gotículas e partículas de gelo e altera a estrutura de temperatura das nuvens e, também, pode causar derretimento da neve e do gelo devido a sua absorção da radiação solar (Bond et al., 2013).

Quanto aos efeitos à saúde, há associação de variações diárias na concentração de $\mathrm{BC}$, e também de médio e longo prazo, com mudanças na saúde devido a mortalidade cardiovascular e hospitalizações cardiopulmonares (Janssen et al., 2012). Estudos de efeitos à saúde humana em curto prazo mostram que as associações com BC são mais robustas do que as com $\mathrm{MP}_{2,5}$ ou $\mathrm{MP}_{10}$, sugerindo que o $\mathrm{BC}$ é um melhor indicador de substâncias nocivas de material particulado originado de processos de combustão (em especial veículos) do que somente a concentração em massa do MP (Janssen et al., 2012). Tem sido observado que a quantidade de $\mathrm{BC}$ presente no $\mathrm{MP}_{2,5}$ é fortemente dependente da distância das vias de intenso tráfico veicular, com diminuição significativa das concentrações até $100 \mathrm{~m}$ de distância (Ranft et al., 2009).

$\mathrm{Na}$ maioria das áreas urbanas do Brasil os veículos são considerados a principal fonte de emissão de poluentes na atmosfera. Quase 21 milhões de pessoas vivem na RMSP, dentre esses aproximadamente 12 milhões moram na capital (IBGE, 2015) e a frota veicular, principal fonte de poluição da região, ultrapassa 7 milhões de veículos (Carvalho et al., 2015; CETESB, 2015). Experimentos realizados em túneis rodoviários são importantes na avaliação de fatores de emissão de poluentes pela frota real circulando na região de estudo, o que também evita efeitos meteorológicos e das transformações fotoquímicas. $\mathrm{Na}$ RMSP, observou-se diminuição dos fatores de emissão de $\mathrm{MP}_{2,5}$ entre os anos de 2004 e 2011, obtidos em experimentos de túneis (Sánchez-Ccoyllo et al., 2009; PerezMartinez et al., 2014). Porém, a emissão por veículos pesados (movidos a diesel) diminui na metade, enquanto que as emissões por veículos leves diminuíram quase 5 vezes neste período, ou seja, os veículos pesados continuam sendo a principal fonte de $\mathrm{MP}_{2,5}$. A contribuição de veículos pesados nas emissões de BC foi 29 vezes maior que a de veículos leves em 2004 e, provavelmente, pode ser relativamente ainda mais alta, considerando que as emissões de MP por veículos leves têm diminuído mais significativamente (Sánchez-Ccoyllo et al., 2009; Perez-Martinez et al., 2014).

Outros estudos na RMSP mostraram que mais de 90\% do BC está presente no MP fino, apresentando máximos de concentração em massa na menor fração ( $\mathrm{d}<0,094 \mu \mathrm{m})$, e um pico secundário na moda de acumulação $(\mathrm{d} \sim 0,59 \mu \mathrm{m})$, indicando que o BC foi originado de emissões primárias como partículas ultrafinas (Ynoue et al., 2004). Também foi observado que a proporção em massa de $\mathrm{BC}$ presente no $\mathrm{MP}_{2,5}$ foi de $21 \pm 4 \%$ no inverno e $28 \pm 10 \%$ no verão, com concentração média de $\mathrm{BC}$, respectivamente, de $7,6 \mu \mathrm{g} / \mathrm{m}^{3}$ e $4,1 \mu \mathrm{g} / \mathrm{m}^{3}$ (Castanho e Artaxo, 2001). Mais recentemente observou-se aumento nesses valores, atingindo a proporção de $38 \pm 14 \%$ e a concentração média de BC de 10,6 $\pm 6,4 \mu \mathrm{g} / \mathrm{m}^{3}$ (Miranda et al., 2012). Comparando-se seis capitais do Brasil (São Paulo, Rio de Janeiro, Curitiba, Porto Alegre, Belo Horizonte e Recife), São Paulo foi a que apresentou maiores valores de $\mathrm{BC}$ presente no $\mathrm{MP}_{2,5}$ (Miranda et al., 2012). 
Conforme o resultado de vários estudos, o BC passou a ser considerado um marcador de fonte veicular para a RMSP, tornando-se um parâmetro para analisar os efeitos das partículas advindas dos veículos e seus efeitos na saúde. Os efeitos da poluição do ar sobre a saúde são amplos, principalmente sobre os sistemas respiratório e cardiovascular. Toda população é afetada, alguns mais que outros, e pode variar com as condições de saúde ou idade. As pessoas mais suscetíveis aos efeitos dos poluentes do ar são separadas em grupos de risco, que são constituídos por crianças, idosos, grávidas, pobres e pessoas com doenças pré-existentes (Peled, 2011). Dentre todos os poluentes o MP é o que afeta mais pessoas, tanto em exposições de curto prazo como de longo prazo (WHO, 2005). Estudos sugerem que o MP causa maior risco relativo aos idosos (Gouveia e Fletcher, 2000; Fischer et al., 2003) devido ao seu sistema imunológico debilitado, acumulação de substâncias tóxicas com o tempo e doenças pré-existentes (Sun e Gu, 2008).

Estudo realizado em seis capitais brasileiras mostrou que São Paulo foi a região metropolitana com mais alta estimativa de número de mortes associadas ao $\mathrm{MP}_{2,5}$ (Miranda et al., 2012). Como mencionado anteriormente, um dos componentes do $\mathrm{MP}_{2,5}$ que pode causar sérios danos à saúde é o BC, principalmente para idosos. A exposição de idosos ao BC é significativamente associada com distúrbios do controle autonômico do coração (Schwartz et al., 2005), aumento na pressão arterial (Mordukhovich et al., 2009), declínio cognitivo (Power et al., 2011) e com a piora de quadros de doenças pré-existentes como doença cardíaca coronária e diabetes (Fang et al., 2012). No Brasil não há estudos sobre o efeito do BC na saúde.

A população idosa cresce significativamente, sendo o segmento de 80 anos ou mais, o que registra o maior crescimento (Alessandri e Maeda, 2011), portanto o que requer maiores estudos sobre o efeito da poluição do ar. Contudo, antes disso, é preciso conhecer melhor o ar que essa população está respirando. Acredita-se que a mistura complexa de componentes químicos e elementares que compõem o MP é, em parte, responsável pela sua toxicidade (Brokamp et al., 2015). A exposição aos poluentes baseada em estimativas da residência pode ser uma excelente medida de exposição pessoal em pessoas aposentadas (Power et al., 2011), como a maioria dos idosos brasileiros que não estão mais expostos ao ambiente de trabalho, e sim, aos ambientes domiciliares.

A maioria dos estudos utilizam a concentração em massa de $\mathrm{MP}_{2,5}$ ao ar livre em locais de monitoramento como um substituto para a exposição pessoal. Contudo, Brokamp et al. (2015) mostraram que a composição elementar de $\mathrm{MP}_{2,5}$ obtido em estações de monitoramento não tem precisão para representar a exposição pessoal ao $\mathrm{MP}_{2,5}$.

A partir do acima exposto, nosso estudo visa avaliar a qualidade do ar de residências de idosos na RMSP, com destaque para o Black Carbon presente em partículas mais finas $\left(\mathrm{MP}_{0,25}\right)$. Destacamos que este trabalho é pioneiro no
Brasil, seja pela amostragem em residências de idosos, que no desenvolver do Projeto Temático, descrito a seguir na seção 2, também realizaram testes de cognição e equilíbrio em câmara climatizada a $16{ }^{\circ} \mathrm{C}$ (frio), $24^{\circ} \mathrm{C}$ (conforto) e $32{ }^{\circ} \mathrm{C}$ (calor) na Faculdade Politécnica da USP. Além destes testes e coletas, foi feita avaliação de composição química do $\mathrm{MP}_{0,25}$, que tem alto potencial de danos à saúde por penetrar mais profundamente no trato respiratório. Nossos resultados podem, em conjunto com informações de qualidade do ar externo obtidos por rede oficial de monitoramento, nortear políticas públicas visando melhoria da qualidade de vida dos idosos das grandes cidades brasileiras.

\section{Materiais e Métodos}

Esse estudo faz parte de um amplo projeto temático intitulado "Biometeorologia humana: análise dos efeitos de variáveis ambientais (meteorológicas, conforto térmico e poluição atmosférica) e das mudanças climáticas na população geriátrica da cidade de São Paulo" (FAPESP 2010/10189-5) envolvendo interdisciplinaridade de várias áreas. As amostragens de MP deste trabalho foram efetuadas em residências de idosos voluntários participantes deste projeto temático, com o seu consentimento e aprovação pelo comitê de ética (número de parecer 619.274).

Os critérios utilizados para seleção dos idosos incluem: ter mais de 60 anos, de acordo com critério pré-estabelecido pelos médicos geriatras do Projeto Temático, não necessitar de uso de muletas ou andador, não ser obeso, não estar tomando remédio para depressão, ter escolaridade maior ou igual a 4 anos e ser aprovado em todos os testes realizados em nossa triagem. Esses testes são o MineExame do Estado Mental, MEEM - (Folstein et al., 1975) a fim de avaliar algum déficit cognitivo do indivíduo, a Escala de Depressão Geriátrica, EDG - (Sheikh et al., 1991) para avaliar possíveis sintomas de depressão e o Short Physical Performance Battery, SPPB - (Guralnik et al., 1994) a fim de verificar previamente déficit de equilíbrio.

Para a amostragem de MP utilizamos impactador em cascata de uso pessoal denominado Personal Cascate Impactor Sampler (PCIS - SKC Cat. N ${ }^{0}$ 225-370). Esse aparelho é um impactador em cascata miniaturizado que permite a separação das partículas suspensas no ar em vários intervalos de tamanho e é acoplado a uma bomba Leland Legacy® (SKC Cat. No. 100-3002) com taxa de fluxo de 9 L/min (Misra et al., 2002; Sioutas, 2004; Singh et al., 2003). No PCIS as partículas são separadas nos seguintes diâmetros aerodinâmicos: 10 a 2,5; 2,5 a 1,0; 1,0 a 0,$5 ; 0,5$ a 0,25 e d < 0,25 $\mu \mathrm{m}$ (Sioutas, 2004) e são usados filtros de Teflon de 2,5 centímetros de diâmetro. O Teflon é eficiente no recolhimento de partículas acima do ponto de corte de cada etapa e não recolhem excessivamente partículas abaixo do ponto de corte, além de poderem ser usados 
para a análise gravimétrica e de composição química (Misra et al., 2002).

As amostragens foram realizadas de maio de 2014 a julho de 2015, uma única vez em cada residência por 24 h, com exceção de uma casa onde dois idosos residentes foram voluntários no projeto. A concentração em massa de MP nas amostras foi quantificada por gravimetria com balança de precisão de $1 \mu \mathrm{g}$ (MX5; Mettler-Toledo, Columbus, OH, USA) em sala com ambiente controlado $\left(22 \pm 2{ }^{\circ} \mathrm{C}\right.$ e $45 \pm 3 \%$ de UR). A concentração em massa de $\mathrm{MP}_{2,5}$ foi calculada a partir da soma das concentrações de MP nos quatro últimos estágios de impactação do PCIS (2,5 a 1,$0 ; 1,0$ a 0,$5 ; 0,5$ a 0,25 e d $<0,25 \mu \mathrm{m}$ ).

Uma vez que as partículas finas atingem os alvéolos pulmonares (Arbex et al., 2012) e as ultrafinas podem translocar para outros órgãos (Kreyling et al., 2006) optamos por analisar o $\mathrm{BC}$ nas partículas mais finas amostradas pelo PCIS, as partículas menores que $0,25 \mu \mathrm{m}\left(\mathrm{MP}_{0,25}\right)$. A concentração de massa $\mathrm{BC}$ foi quantificada através de medidas de refletância ótica com um refletômetro (smoke stain reflectometer modelo 43D; Difusão Systems Ltd, Londres, Reino Unido). A partir destas medidas, a concentração do $\mathrm{BC}\left(\mathrm{em} \mu \mathrm{g} / \mathrm{m}^{3}\right)$ foi calculada pela Eq. (1), válida para o equipamento usado para nossas medidas (Miranda et al., 2012):

$$
\begin{aligned}
B C & =((82,794-(73,206 \log (m)) \\
& \left.\left.+15,901 \log (m)^{2}\right) a\right) / v
\end{aligned}
$$

onde $m$ é a média de duas medidas de refletância realizadas em um mesmo filtro; $a$ é a área do filtro em que há partículas (área amostrada) $\left[\mathrm{cm}^{2}\right] ; v$ é o volume de ar amostrado $\left[\mathrm{m}^{3}\right]$.

Na literatura, a terminologia Black Carbon é a utilizada para medidas diferentes que variam conforme suas propriedades, técnicas de medição e incertezas relacionadas, o que pode causar divergência de resultados, confusão na comparação entre trabalhos científicos e no uso do termo na legislação de qualidade do ar. Em virtude destes fatos, Petzold et al. (2013) recomendam terminologias adequadas para esclarecer os termos usados para BC. Em nosso trabalho usaremos medidas obtidas através de técnica de refletância e, portanto, usaremos a terminologia $r B C$ doravante, de acordo com Petzold et al. (2013), e faremos comparações com trabalhos que usaram a mesma técnica de medição.

Escolhemos 10\% das amostras (seis dias) com maior proporção de $\mathrm{rBC}$ no $\mathrm{MP}_{0,25}$ para analisarmos as condições meteorológicas que possam ter influenciado essa maior proporção de rBC nessas amostras. Para tal, usamos dados de temperatura, UR, precipitação e velocidade do vento do dia de amostragem, obtidos da Estação Meteorológica do Instituto de Astronomia, Geofísica e Ciências Atmosféricas da Universidade de São Paulo (IAG-USP), localizada no bairro da Água Funda.

\section{Resultados e Discussões}

Durante a triagem foram aprovados 77 idosos e desses 63 concordaram em participar deste estudo. Deste conjunto, três apresentaram problemas de amostragem e por isso, ao final, foram consideradas válidas 60 amostras. Dentre essas 60 residências de idosos amostradas, duas foram na cidade de Osasco, uma em Embu das Artes e o restante na cidade de São Paulo. A Fig. 1 mostra a localização dessas residências na RMSP. As regiões de São Paulo com maior concentração de idosos participantes do projeto são as mesmas regiões onde há maior proporção de idosos com relação ao número de crianças, principalmente de idosos mais velhos (Alessandri e Maeda, 2011).

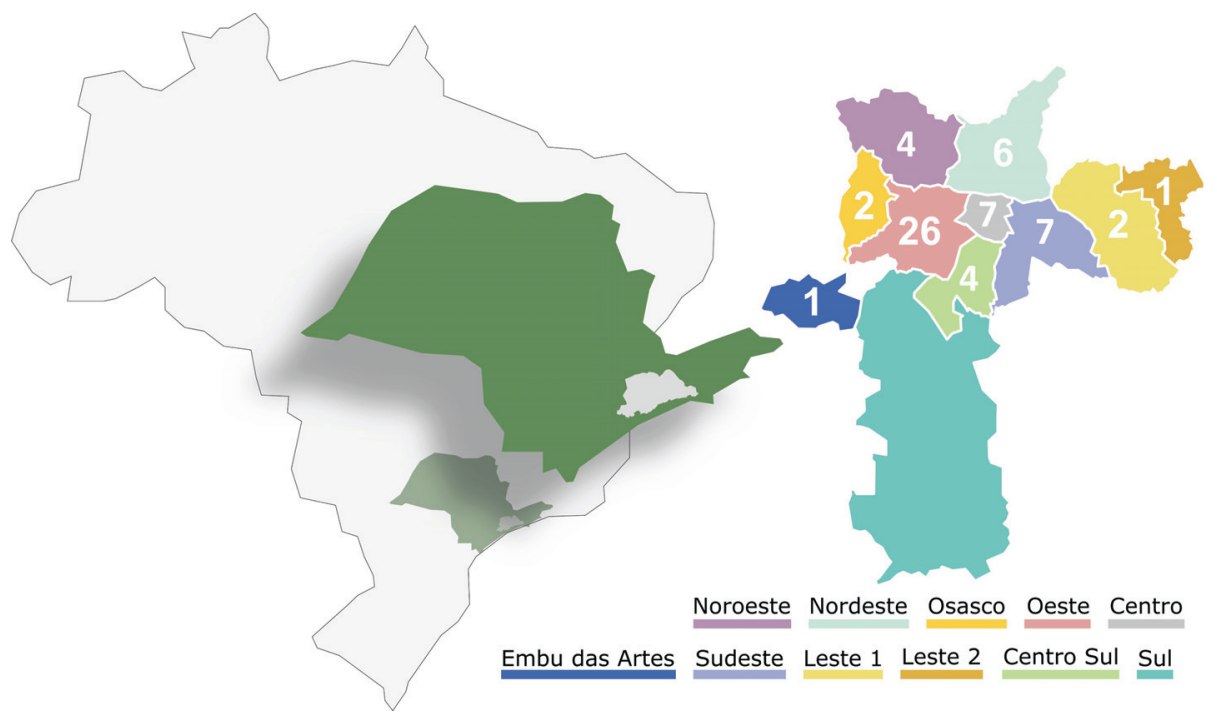

Figura 1 - Localização da região de estudo com destaque para as cidades de São Paulo, Osasco e Embu das Artes com seus respectivos números de amostragens em residência de idosos na Região Metropolitana de São Paulo, Estado de São Paulo, Brasil. 
Por volta de 49,5\% do $\mathrm{MP}_{2,5}$ amostrado nas residências era composto por $\mathrm{MP}_{0,25}$ (Fig. 2). A média de $\mathrm{MP}_{0,25}$ nas residências foi de $13,6 \pm 25,7 \mu \mathrm{g} / \mathrm{m}^{3}$ (altos valores de desvio padrão são devido a um caso extremo) e a de rBC foi de $2,8 \pm 2,3 \mu \mathrm{g} / \mathrm{m}^{3}$. A maior concentração de $\mathrm{MP}_{0,25}$ foi de $203,5 \mu \mathrm{g} / \mathrm{m}^{3}$ (Fig. 3b) esse valor extremamente alto deve-se ao fato de que no período de amostragem havia obras de construção civil a cerca de $50 \mathrm{~m}$ da residência do idoso. Destaca-se que esta amostra apresentou a menor proporção de $\mathrm{rBC}(0,9 \%)$ com $1,81 \mu \mathrm{g} / \mathrm{m}^{3}$ (Fig. 3b). Após 6 meses fizemos uma nova amostragem com o PCIS (amostra 43) na mesma residência da amostragem 27 (Fig. 3b), mas referente a outro idoso morador do local. A concentração em massa de $\mathrm{MP}_{0,25}$ e a proporção de $\mathrm{rBC}$ no $\mathrm{MP}_{0,25}$ foram bem diferentes, com $4,8 \mu \mathrm{g} / \mathrm{m}^{3}$ e $27,7 \%$, respectivamente. Isso reforça a influência da obra de construção na amostragem inicial (amostra 27). Na Fig. 4 são apresentadas as imagens dos filtros amostrados contendo baixa (Fig. 4a) e alta (Fig. 4b) concentração de Black Carbon.

Atualmente não há limiar padrão nacional, nem internacional, para $\mathrm{MP}_{0,25}$, e medidas externas de partículas tão pequenas não têm sido feitas por órgão reguladores, como a CETESB no estado de São Paulo. Apesar deste fato, o $\mathrm{MP}_{0,25}$ ultrapassou a recomendação da Organização Mundial da Saúde (OMS) para $\mathrm{MP}_{2,5}$ em 11,7\% das residências;

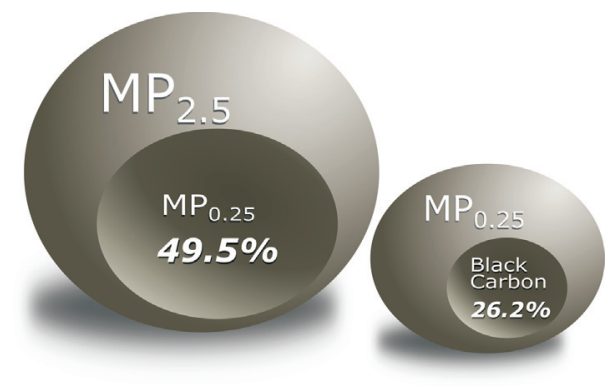

Figura 2 - Proporção de concentração de $\mathrm{MP}_{0,25}$ no $\mathrm{MP}_{2,5}$ (à esquerda) e proporção de Black Carbon no $\mathrm{MP}_{0,25}$ (à direita) medido nas amostragens de $24 \mathrm{~h}$ realizados nas residências de idosos na RMSP. lembrando que o $\mathrm{MP}_{0,25}$ está presente no $\mathrm{MP}_{2,5}$. Essa alta concentração de partículas mais finas é extremamente perigosa à saúde, principalmente dos idosos.

$\mathrm{O}$ BC também não é um poluente regulamentado e monitorado, mas pode apresentar valores preocupantes. A maior concentração de $\mathrm{rBC}$ foi de $13,1 \mu \mathrm{g} / \mathrm{m}^{3}$ (Fig. 3a) devido à ausência de chuvas dias antes da amostragem, e durante a mesma, UR baixa e ventos fracos, além de a residência estar próxima a vias de tráfico intenso (amostra 6, Tabela 1). Por volta de $26,2 \pm 12,3 \%$ da massa do $\mathrm{MP}_{0,25}$ amostrado nas residências foi composta por rBC (Fig. 2). Estudos ao ar livre na RMSP mostraram proporções que podem variar de $21-38 \%$ de $\mathrm{rBC}$ nas amostras de $\mathrm{MP}_{2,5}$ (Castanho e Artaxo, 2001; Ynoue e Andrade, 2004; Miranda et al., 2012). Não há análises de $\mathrm{BC}$ em $\mathrm{MP}_{0,25} \mathrm{em}$ ambiente interno e externo no Brasil, tão pouco em outros países. Em 13,3\% das residências, o $\mathrm{rBC}$ presente no $\mathrm{MP}_{0,25}$ excedeu $40 \%$ de sua composição, isso se deve ao fato de que todas essas residências estão localizadas em grandes avenidas ou próximas a elas, com fluxo veicular intenso e
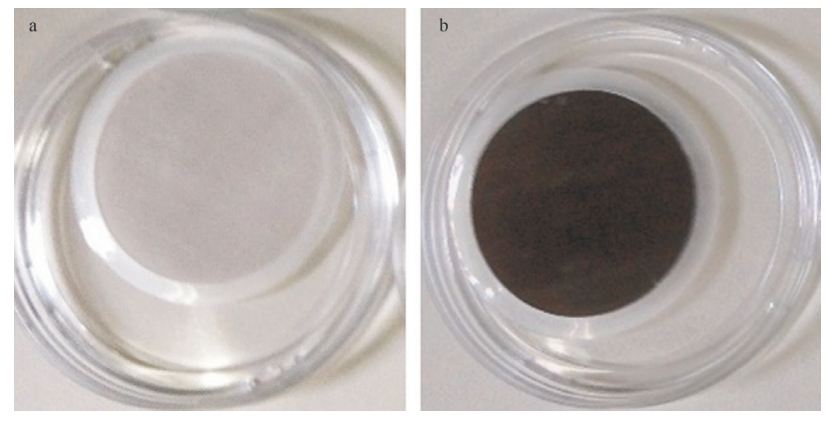

Figura 4 - Exemplos da aparência do filtro do $\mathrm{MP}_{0,25}$ após amostragem nas residências de idosos na Região Metropolitana de São Paulo. A foto da esquerda (a) mostra o filtro de residência mais distante de vias de trafego intenso, além de ocorrência de muita chuva, indicando baixa concentração em massa de Black Carbon (rBC) e a foto da direita (b) é um filtro de local próximo a via de trafego intenso em dia sem chuva, indicando alta concentração em massa de rBC.

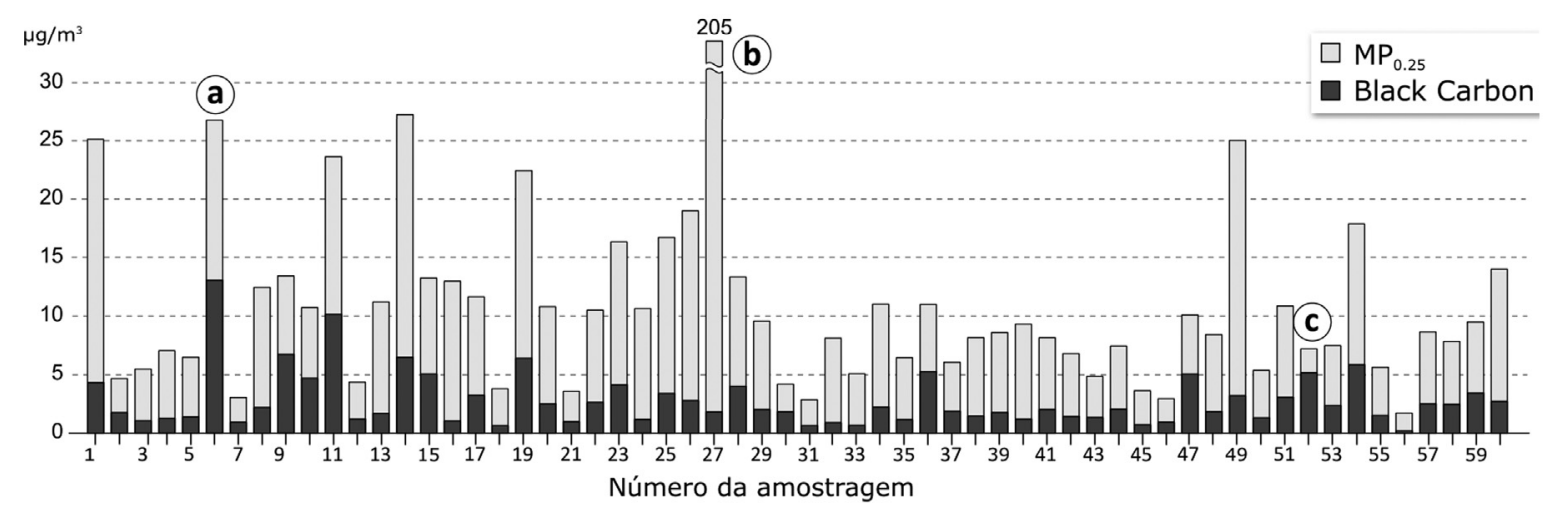

Figura 3 - Concentração em massa de $\mathrm{MP}_{0,25}$ (total cinza mais preto) das amostragens de $24 \mathrm{~h}$ realizadas nas residências de idosos, e o rBC medido nessas amostras (em preto). A amostragem 6 (letra a) representa a maior concentração em massa de rBC, a amostragem 27 (letra b) o maior valor de massa de $\mathrm{MP}_{0,25}$ e a amostragem 52 (letra c) a maior proporção de $\mathrm{rBC}$ no $\mathrm{MP}_{0,25}$. 
Tabela 1 - Tabela com os dados meteorológicos na estação do IAG - USP para as amostragens com maior proporção de rBC no MP ${ }_{0.25}$ (10\% das amostras). As colunas apresentam precipitação (P), dias sem precipitação antes do início da amostragem (S/P), umidade relativa (UR), velocidade do vento (V) e temperatura do ar (T), referentes ao dia de início (i) e dia de fim (f) das amostragens de $24 \mathrm{~h}$.

\begin{tabular}{|c|c|c|c|c|c|c|c|c|c|c|c|c|}
\hline Amostra & Data & $\mathrm{rBC}(\%)$ & Grandes avenidas & Pi (mm) & $\operatorname{Pf}(\mathrm{mm})$ & $\mathrm{S} / \mathrm{P}$ (dias) & $\mathrm{UR}_{\mathrm{i}}(\%)$ & $\mathrm{UR}_{\mathrm{f}}(\%)$ & $\mathrm{V}_{\mathrm{i}}(\mathrm{m} / \mathrm{s})$ & $\mathrm{Vf}(\mathrm{m} / \mathrm{s})$ & $\operatorname{Ti}\left({ }^{\circ} \mathrm{C}\right)$ & $\operatorname{Tf}\left({ }^{\circ} \mathrm{C}\right)$ \\
\hline 30 & $26 / 11 / 2014$ & 44 & $\begin{array}{l}\text { Bandeirantes e } \\
\text { Ibirapuera }\end{array}$ & 42 & 0,3 & 0 & 90 & 77 & 6,4 & 5 & 22,2 & 20,6 \\
\hline 36 & $26 / 03 / 2015$ & 48 & Jaguaré & 0 & 0 & 3 & 72 & 76 & 5,2 & 2,9 & 22,8 & 22 \\
\hline 6 & $01 / 07 / 2014$ & 49 & Marginal & 0 & 0 & 4 & 56 & 71 & 2,5 & 2,5 & 15,2 & 16,6 \\
\hline 47 & $19 / 05 / 2015$ & 50 & $\begin{array}{l}\text { Professor Castro } \\
\text { Junior e Conceição }\end{array}$ & 0 & 0 & 5 & 88 & 86 & 3,5 & 5,3 & 18,1 & 17,9 \\
\hline 9 & $26 / 06 / 2014$ & 50 & $\begin{array}{c}\text { Dom Pedro I e Av. } \\
\text { do Estado }\end{array}$ & 0,1 & 0 & 0 & 74 & 76 & 3,3 & 3,5 & 18,2 & 19,1 \\
\hline 52 & $10 / 06 / 2015$ & 72 & $\begin{array}{c}\text { Brigadeiro Faria } \\
\text { Lima e Nações } \\
\text { Unidas }\end{array}$ & 0 & 0 & 0 & 73 & 62 & 3 & 6,2 & 18,4 & 20,6 \\
\hline
\end{tabular}

constante. Em uma dessas residências a porcentagem de rBC na amostra chegou a 71,6\% do total da massa (Fig. 3c).

Os dias com mais altas proporções de $\mathrm{rBC}$ no $\mathrm{MP}_{0,25}$ das residências foram os dias das amostragens 6, 9, 30, 36, 47 e 52 e são apresentados na Tabela 1, juntamente com dados meteorológicos e nome das grandes avenidas próximas. A velocidade do vento, UR e temperatura não apresentaram padrão específico para esses testes. Houve precipitação somente durante as amostragens 9 e 30. Durante a amostragem 30 ocorreu intensa precipitação e, em virtude disso, apresentou baixas concentrações de $\mathrm{MP}_{0,25}$. Contudo, a proporção de $\mathrm{rBC}$ na amostra esteve alta, pois a residência fica muito próxima de duas grandes avenidas com intenso fluxo veicular, a Avenida Bandeirantes e Avenida Ibirapuera. A amostra 9 apresentou características semelhantes a 30, apesar de ter ocorrido $0,1 \mathrm{~mm}$ de precipitação, também é uma residência entre duas avenidas de intenso tráfego de veículos (Avenida Dom Pedro I e Avenida do Estado). Nas demais amostragens não houve precipitação, mas também são residências próximas de vias de tráfego intenso, o que justificaria altas proporções de rBC observadas no $\mathrm{MP}_{0,25}$.

\section{Conclusões}

Atualmente não há análises de $\mathrm{BC}$ em $\mathrm{MP}_{0,25} \mathrm{em}$ ambiente interno e externo no Brasil, tão pouco em outros países, não havendo seu monitoramento por órgãos governamentais, nacional ou internacional. Logo, não existem recomendações da OMS, ou outro órgão, para $\mathrm{MP}_{0,25}$ e BC. Apesar disso, o $\mathrm{MP}_{0,25}$ amostrado nas residências dos idosos na RMSP ultrapassou a recomendação da OMS para $\mathrm{MP}_{2,5}$ em 11,7\% das residências. Essa alta concentração de partículas mais finas é extremamente perigosa à saúde, principalmente dos idosos. $\mathrm{O}$ rBC também apresentou valores preocupantes, compondo em média $26,2 \%$ da massa do $\mathrm{MP}_{0,25}$ amostrado nas residências dos idosos. Em 13,3\% das residências, o rBC excedeu $40 \%$ da composição do $\mathrm{MP}_{0,25}$, isso se deve ao fato de que essas residências estão localizadas próximas a grandes avenidas com fluxo veicular intenso e constante, incluindo ônibus e caminhões de grande porte. Uma vez que o BC é um marcador de tráfego veicular que pode ter altas concentrações residenciais e é comprovadamente danoso à saúde, torna-se útil e necessário o uso do BC como um indicador adicional na avaliação de ações locais que tenham o intuito de reduzir a exposição da população às partículas geradas por combustão, principalmente advindas de veículos, fonte predominante da poluição do ar na RMSP.

\section{Agradecimentos}

Ao CNPq, FAPESP e CAPES (PROEX - Programa de Pós-Graduação em Meteorologia, IAG-USP) por terem possibilitado e financiado esta pesquisa e ao Kaonan Micadei e Gavin Roy pelas discussões.

\section{Referências}

ALESSANDRI, M.R.; MAEDA, M.D. Informes Urbanos: Cresce o número de idosos. Prefeitura deSão Paulo, Secretaria Municipal de Desenvolvimento Urbano de São Paulo, p. $1-3$, 2011. http://smdu.prefeitura.sp.gov.br/informes_urbanos/pdf/5.p df.

ANDRADE, M.F.; MIRANDA, R.M.; FORNARO, A.; KERR, A.; OYAMA, B.; ANDRE, P.A.; SALDIVA, P. Vehicle emissions and $\mathrm{PM}_{2.5}$ mass concentrations in six Brazilian cities, Air Quality, Atmosphere \& Health, v. 5, n. 1, p. 79-88, 2012.

ARBEX, M.A.; SANTOS, U.D.P.; MARTINS, L.C.; SALDIVA, P.H.N.; PEREIRA, L.A.A.; BRAGA, A. L. F. Air pollution and the respiratory system, Jornal Brasileiro de Pneumologia, v. 38, n. 5, p. 643-655, 2012.

BOND, T.C.; DOHERTY, S.J.; FAHEY, D.W.; FORSTER, P.M.; BERNTSEN, T.; DEANGELO, B.J.; FLANNER, M.G.; GHAN, S.; KÄRCHER, B.; KOCH, D.; KINNE, S.; KONDO, Y.; QUINN, P.K.; SAROFIM, M.C.; SCHULTZ, M.G.; SCHULZ, M.; VENKATARAMAN, C.; ZHANG, H.; ZHANG, S.; BELLOUIN, N.; GUTTIKUNDA, S. K.; HOPKE, P.K.; JACOBSON, M.Z.; KAISER, J.W.; KLI- 
MONT, Z.; LOHMANN, U.; SCHWARZ, J.P.; SHINDELL, D.; STORELVMO, T.; WARREN, S.G.; ZENDER, C.S. Bounding the role of black carbon in the climate system: A scientific assessment, Journal of Geophysical Research: Atmospheres, v. 118, n. 11, p. 5380-5552, 2013.

BROKAMP, C.; RAO, M.B.; FAN, Z.T.; RYAN, P.H. Does the elemental composition of indoor and outdoor $\mathrm{PM}_{2.5}$ accurately represent the elemental composition of personal $\mathrm{PM}_{2.5}$ ? Atmospheric Environment, v. 101, p. 226-234, 2015.

CETESB. Qualidade do ar no Estado de São Paulo, 2014. Série Relatórios, Secretaria do Estado do Meio Ambiente, São Paulo, p. 1-136, 2015, ISSN 0103-4103. http://www.cetesb.sp.gov.br/ar/qualidade-do-ar/31-publica coes-e-relatorios.

FANG, S.C.; MEHTA, A.J.; ALEXEEFF, S.E.; GRYPARIS, A.; COULL, B.; VOKONAS, P.; CHRISTIANI, D.C.; SCHWARTZ, J. Residential black carbon exposure and circulating markers of systemic inflammation in elderly males: the normative aging study, Environmental Health Perspectives, v. 120, n. 5, p. 674-680, 2012.

FISCHER, P.; HOEK, G.; BRUNEKREEF, B.; VERHOEFF, A.; VAN WIJNEN, J. Air pollution and mortality in the Netherlands: are the elderly more at risk? European Respiratory Journal, v. 21, p. 34S-38S, 2003.

FOLSTEIN, M.F.; FOLSTEIN, S.E.; MCHUGH, P.R. "Minimental state": a practical method for grading the cognitive state of patients for the clinician, Journal of Psychiatric Research, v. 12, n. 3, p. 189-198, 1975.

GOUVEIA, N.; FLETCHER, T. Time series analysis of air pollution and mortality: effects by cause, age and socioeconomic status. Journal of epidemiology and community health, v. 54(10), p. 750-755, 2000.

GURALNIK, J.M.; SIMONSICK, E.M.; FERRUCCI, L.; GLYNN, R.J.; BERKMAN, L.F.; BLAZER, D.G.; SCHERR P.A.; WALLACE, R.B. A short physical performance battery assessing lower extremity function: association with self-reported disability and prediction of mortality and nursing home admission, Journal of Gerontology, v. 49, n. 2, p. M85-M94, 1994.

IBGE. Estimativas da população residente no Brasil e Unidades da Federação com data de referência de $1^{\circ}$ de julho de 2015 . Instituto Brasileiro de Geografia e Estatística, p. 1-146, 2015.

http://www.ibge.gov.br/home/estatistica/populacao/estimat iva2015/estimativa_dou.shtm

JANSSEN, N.A.; GERLOFS-NIJLAND, M.E.; LANKI, T.; SALONEN, R.O.; CASSEE, F.; HOEK, G.; FISHER, P.; BRUNEKREEF, B.; KRZYZANOWSKI, M. Health effects of black. Copenhagen: WHO Regional Office for Europe, p. 1-86, 2012.

KREYLING, W.G.; SEMMLER-BEHNKE, M.; MÖLLER, W. Ultrafine particle-lung interactions: does size matter? Journal of Aerosol Medicine, v. 19, n. 1, p. 74-83, 2006.

MARTINS, L.D.; MARTINS, J.A.; FREITAS, E.D.; MAZZOLI, C.R.; GONÇALVES, F.L.T.; YNOUE, R.Y.; HALLAK R.; ALBUQUERQUE T.T.A.; ANDRADE, M.F. Potential health impact of ultrafine particles under clean and polluted urban atmospheric conditions: a model-based study, Air Quality, Atmosphere \& Health, v. 3, n. 1, p. 29-39, 2010.
MIRANDA, R.M.; ANDRADE, M.F.; FORNARO, A.; ASTOLFO, R.; ANDRE, P.A.; SALDIVA, P. Urban air pollution: a representative survey of $\mathrm{PM}_{2.5}$ mass concentrations in six Brazilian cities, Air Quality, Atmosphere \& Health, v. 5, n. 1, p. 63-77, 2012.

MISRA, C.; SINGH, M.; SHEN, S.; SIOUTAS, C.; HALL, P. Development and evaluation of a personal cascade impactor sampler (PCIS), Journal of Aerosol Science, v. 33, n. 7, p. 1027-1047, 2002.

MORDUKHOVICH, I.; WILKER, E.H.; SUH MACINTOSH, H.H.; WRIGHT, R.O.; SPARROW, D.; VOKONAS, P.S.; SCHWARTZ, J.D. Black carbon exposure, oxidative stress genes, and blood pressure in a repeated-measures study, Environmental Health Perspectives, v. 117, n. 11, p. 17671772, 2009.

PELED, R. Air pollution exposure: Who is at high risk? Atmospheric Environment, v. 45(10), p. 1781-1785, 2011.

PÉREZ-MARTÍNEZ, P.J.; MIRANDA, R.M.; NOGUEIRA, T.; GUARDANI, M.L.; FORNARO, A.; YNOUE, R.; ANDRADE, M.F. Emission factors of air pollutants from vehicles measured inside road tunnels in São Paulo: case study comparison, International Journal of Environmental Science and Technology, v. 11, n. 8, p. 2155-2168, 2014.

PETZOLD, A.; OGREN, J. A.; FIEBIG, M.; LAJ, P.; LI, S.M.; BALTENSPERGER, U.; HOLZER-POPP, T.; KINNE, S.; PAPPALARDO, G.; SUGIMOTO, N.; WEHRLI, C.; WIEDENSOHLER, A.; ZHANG, X.Y. Recommendations for reporting "black carbon" measurements, Atmospheric Chemistry and Physics, v. 13, n. 16, p. 8365-8379, 2013.

POWER, M.C.; WEISSKOPF, M.G.; ALEXEEFF, S.E.; COULL, B.A.; SPIN III, A.; SCHWARTZ, J. Trafficrelated air pollution and cognitive function in a cohort of older men, Environmental Health Perspectives, v. 119, n. 5, p. 682-687, 2011

RANFT, U.; SCHIKOWSKI, T.; SUGIRI, D.; KRUTMANN, J.; KRÄMER, U. Long-term exposure to traffic-related particulate matter impairs cognitive function in the elderly, Environmental Research, v. 109(8), p. 1004-1011, 2009.

SÁNCHEZ-CCOYLLO, O.R.; YNOUE, R.Y.; MARTINS, L.D.; ASTOLFO, R.; MIRANDA, R. M.; FREITAS, E.D.; MOREIRA, A.; ANDRADE, M.F. Vehicular particulate matter emissions in road tunnels in Sao Paulo, Brazil, Environmental Monitoring and Assessment, v. 149, n. 1-4, p. 241-249, 2009.

SHEIKH, J.I.; YESAVAGE, J.A.; BROOKS, J.O.; FRIEDMAN, L.; GRATZINGER, P.; HILL, R.D.; ZADEIK, A.; CROOK, T. Proposed factor structure of the Geriatric Depression Scale, International Psychogeriatrics, v. 3, n. 1, p. 23-28, 1991.

SINGH, M.; MISRA, C.; SIOUTAS, C. Field evaluation of a personal cascade impactor sampler (PCIS), Atmospheric Environment, v. 37, n. 34, p. 4781-4793, 2003.

SIOUTAS, C. Development of New Generation Personal Monitors for Fine Particulate Matter (PM) and its Metal Content, Mickey Leland National Urban Air Toxics Research Center, NUATRC Research Report, v. 2, 2004.

SCHWARTZ, J.; LITONJUA, A.; SUH, H.; VERRIER, M.; ZANOBETTI, A.; SYRING, M.; NEARING, B.; VERRIER, R.; STONE, P.; MACCALLUM, G.; SPEIZER, F.E.; GOLD, D.R. Traffic related pollution and heart rate variabil- 
ity in a panel of elderly subjects, Thorax, v. 60, n. 6, p. $455-461,2005$.

SUN, R.; GU, D. Air pollution, economic development of communities, and health status among the elderly in urban China, American Journal of Epidemiology, v. 168, n. 11, p. 1311-1318, 2008.

YNOUE, R.Y.; ANDRADE, M.F. Size-resolved mass balance of aerosol particles over the São Paulo metropolitan area of
Brazil, Aerosol Science and Technology, v. 38, n. S2, p. 52-62, 2004.

WHO - World Health Organization. Air Quality Guidelines Global Update 2005: Report on a Working Group Meeting. WHO Regional Office for Europe, p. 1-30, 2005. http://www.euro.who.int/_data/assets/pdf_file/0008/147851/E87950.pdf.

All the contents of this journal, except where otherwise noted, is licensed under a Creative Commons Attribution License CC-BY. 